\title{
Synthesis and biochemical characterization of silver nanoparticles grafted chitosan (Chi-Ag-NPs): in vitro studies on antioxidant and antibacterial applications
}

\author{
Pavan Kumar Dara' · R. Mahadevan ${ }^{1}$ - P. A. Digita ${ }^{2}$ - S. Visnuvinayagam ${ }^{1}$ - Lekshmi R. G. Kumar ${ }^{1}$ - Suseela Mathew ${ }^{1}$. \\ C. N. Ravishankar ${ }^{1} \cdot$ R. Anandan ${ }^{1}$ (I)
}

Received: 19 September 2019 / Accepted: 13 February 2020 / Published online: 16 March 2020

(c) Springer Nature Switzerland AG 2020

\begin{abstract}
Metal-based nanoparticles with potential antioxidant and antimicrobial properties have led to their incorporation of biomaterials for human health care importance. The present investigation is to study on the silver nanoparticles grafted chitosan (Chi-Ag-NPs) composite materials due to their potential applications. Chi-Ag-NPs were synthesized by chemical reduction of silver nitrate salts and biodegradable chitosan. The obtained Chi-Ag-NPs were characterised for Fouriertransform infrared spectroscopy (FTIR), scanning electron microscope (SEM), Transmission electron microscopy (TEM), $\mathrm{X}$-ray powder diffraction, antioxidant and antibacterial properties. UV-visible spectra showed the configuration proficiency of Ag-NPs and chitosan. FTIR spectra indicated that the functional groups of chitosan have particular interactions with Ag-NPs. Particle size and TEM analyses showed that Chi-Ag-NPs were equivalently dispersed in matrix with an average size of 10-230 $\mathrm{nm}$. The surface morphological structures of chitosan has played important role in influencing the adsorption of Ag-NPs. Chi-Ag-NPs were tested for their bioactive properties and results exhibited higher antioxidant and antibacterial activities by the addition of Ag-NPs. Chi-Ag-NPs composites were non-toxic to L929 fibroblast cells. The present investigation suggested that chitosan will be a good source for the preparation of bionanocomposite materials and also could be useful in different industrial and biomedical applications.
\end{abstract}

Keywords Chitosan silver nanoparticles $\cdot$ Antioxidant and antimicrobial properties $\cdot$ Biocompatibility

\section{Introduction}

The potential applications of nanoparticles (NPs) have attracted the scientific researchers working in various disciplines of food, pharmacological and biomedical sciences towards nanotechnology. Nanoparticles from metal source such as silver $(\mathrm{Ag})$, gold $(\mathrm{Au})$, copper $(\mathrm{Cu})$, titanium (Ti) and Zinc ( $\mathrm{Zn}$ ) play a vital role in nano material science and technology. Recently, there are more attention towards silver nanoparticles (Ag-NPs) for various nutraceutical and biomedical applications as compared to other groups of metal nanoparticles, due to their remarkable and unique optical, mechanical, catalytic, conductivity, heat transfer, low toxicity, high stability, biocompatibility and effective bactericidal effects [1]. Different types of protocols have been developed for producing silver nanoparticles using physical, chemical and biological interventions. Notably, the chemical reduction process of silver nitrate salt in the presence of an organic solvent or water is considered as the most promising applied technology for the synthesis of colloidal Ag-NPs [2], which is commonly incorporated into biopolymer matrices such as chitosan, cellulose, alginate, etc. for variety of tissue engineering and biomedical applications. Investigations by Venkatesan et al.

$\triangle$ R. Anandan, kranandan@rediffmail.com | ${ }^{1}$ ICAR-Central Institute of Fisheries Technology, Cochin, Kerala 682029 , India. ${ }^{2}$ Department of Bioscience, SNGIST Arts and Science College, Ernakulam, Kerala 683511, India. 
[3] have demonstrated that Ag-NPs incorporated chitosan, alginate and polyvinyl alchohol (PVA) biomaterials are highly efficient in controlling pathogenic microorganisms.

Chitin is the second most abundant polysaccharide present in shells of marine crustaceans, insects, cephalopods endoskeletons and fungi cell wall and chitosan is a deacetylated cationic marine polysaccharide derived from chitin through alkali hydrolysis [4]. The basic co-polymeric structure of chitosan is the sequence of repeated $\beta$ (1-4) linked $\mathrm{N}$-acetyl-D-glucosamine and D-glucosamine units with one amino $\left(\mathrm{NH}_{2}\right)$ group and two hydroxyl $(\mathrm{OH})$ groups [5]. Chitosan is one of the most extensively exploited biopolymers in the fields of pharmaceutical, tissue engineering and biomedical industries due to its biocompatibility, non-antigenicity, biodegradability, non-toxicity, high permeability, antioxidant and antimicrobial properties [6, 7]. Studies by Manukumar et al. [1] have shown that bactericidal thymol loaded chitosan silver nanoparticles exerted excellent hemocompatibility. Silver nanoparticles grafted biopolymer films are effectively utilized in the food processing industry as active packaging materials [8].

In the present study, an attempt has been made to develop an effective process for the synthesis of Ag-NPs grafted chitosan with amplified antioxidant and antibacterial properties. The structural, functional and biochemical characterization of the synthesized nanocomposite material were carried out using a UV-visible spectroscopy, Fourier transform infrared spectroscopy (FTIR), scanning electron microscope (SEM), X-ray diffraction (XRD), transmission electron microscope (TEM). The antimicrobial actions of silver nanoparticles (Ag-NPs) grafted chitosan were evaluated against the pathogenic microorganisms such as Pseudomonas aeruginosa, Escherichia coli, and Staphylococcus aureus (MRSA).

\section{Materials and methods}

Chitosan, 2, 2 Diphenyl-1-picrylhydrazyl (DPPH), iron (III) chloride, linoleic acid, $\beta$-carotene, Tween-80, were purchased from Sigma Aldrich (St. Louis, MO, USA). All other chemicals and reagents used in the present study were either analytical grade reagent (AR) or guaranteed grade reagent (GR).

\subsection{Synthesis of chitosan- silver nanoparticles (Chi-Ag-NPs)}

Chemical reduction method was used for the synthesis of chitosan-silver nanoparticles (Chi-Ag-NPs) using chitosan as reducing and stabilizing agent. The synthesis of Chitosan- Silver nanoparticles (Chi-Ag-NPs) was performed according to the method as described by Kalaivani et al.
[5], with slight modifications. $0.5 \%$ of chitosan was prepared by dissolving in $1 \%$ acetic acid. The chitosan solution was then filtered to obtain homogenous solution. An

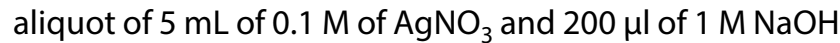
were added to homogenous chitosan solution. $\mathrm{AgNO}_{3}$ and $\mathrm{NaOH}$ were freshly prepared. The chitosan solution was stirred for $6 \mathrm{~h}$ at $90^{\circ} \mathrm{C}$. The colourless chitosan solution was changed to light yellow and then to yellowish brown which indicates the synthesis of Chi-Ag-NPs.

\subsection{Characterization of Chi-Ag-NPs}

\subsubsection{UV-VIS absorption spectra, particle size and zeta potential of the Chi-Ag-NPs}

The synthesized Chi-Ag-NPs composite solution was filtered using a Whatman filter paper No. 1 and UV-Vis absorption spectra were read in the wavelength range of 300-700 nm using double beam spectrophotometer (Shimadzu, UV-1800, Japan). A plot of absorbance vs wavelength was obtained to get UV-Vis absorption spectra. The particle size, polydispersity index (PDI) and Zeta potential of nanocomposites were determined by dynamic light scattering analyses (DLS) using a Zeta Sizer Nano Series (Malvern, Worcestershire, UK). The zeta potential of the suspension was measured with an angle of $15^{\circ}$ at $25^{\circ} \mathrm{C}$ using a Zeta Sizer Nano Series (Malvern, Worcestershire, UK).

\subsubsection{Fourier transform infrared spectroscopy (FT-IR)}

The FTIR spectra of chitosan and Chi-Ag-NPs were analyzed using FTIR spectrometer (Bruker Optik, GmBH, Germany). 30-50 mg of chitosan and Chi-Ag-NPs composite samples were mixed and macerated with a pinch of potassium bromide using pestle and mortar to obtain a fine powder. Then, the resultant powder was placed in the $13 \mathrm{~mm}$ diameter die and then it was made into a pellet by applying a pressure of $5000-10,000$ psi using a hydraulic press. The obtained pellet was removed from the die and placed in the FT-IR sample holder. FTIR analysis is to analyze the presence of different functional groups as well as changes in the secondary structure of polymer in the region of $4000-500 \mathrm{~cm}^{-1}$ wave numbers at $4 \mathrm{~cm}^{-1}$ resolution.

\subsubsection{SEM}

Structure morphology of Chi-Ag-NPs was determined using a scanning electron microscopy (JEOL JSM-5800 LV, Tokyo, Japan). Chitosan and Chi-Ag-NPs composite samples with thickness of 3-5 mm were fixed with $2.5 \%$ $(\mathrm{v} / \mathrm{v})$ glutaraldehyde in $0.2 \mathrm{M}$ phosphate buffer $(\mathrm{pH} 7.2)$ 
for $12 \mathrm{~h}$. Then the samples were rinsed with distilled water for $1 \mathrm{~h}$ and water was removed by dehydration process in the presence of ethanol with a serial concentration of $50,70,80,90$ and $100 \%(\mathrm{v} / \mathrm{v})$. The resultant dried samples were accumulated on a bronze stub and sputter-coated with gold (Sputter coater SPI Module, West Chester, PA, USA). The samples were observed at an accelerated $20 \mathrm{kV}$ voltage.

\subsubsection{X-ray diffraction (XRD) pattern}

X-ray powder diffraction patterns of chitosan and Chi-AgNPs composites were recorded using Analytical X' Pert PRO $\mathrm{X}$-ray diffractometer. The $\mathrm{X}$-ray source was $\mathrm{Cu}$ Ka radiation $(40 \mathrm{kV}, 80 \mathrm{~mA})$. The XRD patterns of samples were taken in the $2 \theta$ range of $10-80$ in a fixed time mode at room temperature at a scanning rate of $4^{\circ} \mathrm{min}^{-1}$.

\subsubsection{TEM}

The morphology and particle size of synthesized Chi-AgNPs composites was visualized using TEM technique (Ultra High Resolution transmission electron microscope 200 kV, JEOL-2010, Tokyo, Japan). TEM images of the Chi-Ag-NPs composites were obtained by placing a drop of the particle solution on a carbon-coated copper grid and was dried keeping under the lamp.

\subsection{Antioxidant activity}

\subsubsection{Diphenyl-1-picryl hydrazyl activity (DPPH) assay}

The DPPH free radical scavenging activities of synthesized Chi-Ag-NPs were determined according to the method described by Yen and Wu [9]. An aliquot of $1.5 \mathrm{ml}$ of ChiAg-NPs composite solution was added to $1.5 \mathrm{ml}$ of $0.2 \mathrm{mM}$ DPPH in $99.50 \%$ ethanol and mixed well by vortex using cyclo-mixer. The solution was incubated in dark at room temperature for $30 \mathrm{~min}$. The absorbance was read at $517 \mathrm{~nm}$ using double beam UV-visible spectrophotometer. The radical scavenging activity was calculated and expressed in percentage using the following formula:

DPPH free radical scavenging activity $(\%)=1-\frac{\mathrm{Abs}_{\text {Sample }}}{\mathrm{Abs}_{\text {Control }}} \times 100$

\subsubsection{Ferric reducing antioxidant power (FRAP) assay}

The ferric reducing power was determined by the method as described by Oyaizu [10]. $1 \mathrm{ml}$ of synthesized Chi-Ag-NPs composite solution was mixed with $2.5 \mathrm{ml}$ of $0.2 \mathrm{M}$ phosphate buffer $(\mathrm{pH} 6.6)$ and $2.5 \mathrm{ml}$ of
$1 \%(\mathrm{w} / \mathrm{v})$ potassium ferri-cyanide. The resultant mixture was incubated for $30 \mathrm{~min}$ at $50^{\circ} \mathrm{C}$ and the reaction was terminated by adding $2.5 \mathrm{ml}$ of $10 \%(\mathrm{w} / \mathrm{v})$ trichloroacetic acid. An aliquot of $2.5 \mathrm{ml}$ solution from the resultant mixture was added to $0.5 \mathrm{ml}$ of $0.1 \%(\mathrm{w} / \mathrm{v}$ ) ferric chloride solution and $2.5 \mathrm{ml}$ of distilled water. Then the resultant mixture was incubated at room temperature for $10 \mathrm{~min}$ and absorbance was read at $700 \mathrm{~nm}$ using double beam UV-visible spectrophotometer. The higher absorbance of the reaction mixture indicates higher reducing power.

\subsection{3 $\beta$-Carotene-linoleic acid bleaching model system}

$\beta$-Carotene-linoleic acid bleaching assay was performed according to the method as described by Koleva et al. [11]. The $\beta$-carotene/linoleic acid stock solution mixture was prepared by dissolving $0.5 \mathrm{mg}$ of $\beta$-carotene, $20 \mu \mathrm{l}$ of linoleic acid and $200 \mu \mathrm{l}$ of tween- 80 in $1 \mathrm{ml}$ of chloroform. The mixture was incubated at $40^{\circ} \mathrm{C}$ till chloroform was evaporated and then $100 \mathrm{ml}$ of deionized water was added. The resultant mixture was stirred vigorously to form an emulsion. Aliquots of $2.5 \mathrm{ml}$ of $\beta$-carotene/linoleic acid emulsion were added to $0.5 \mathrm{ml}$ of Chi-Ag-NPs composite solution and incubated at $50^{\circ} \mathrm{C}$ for $60 \mathrm{~min}$. The absorbance was recorded at $470 \mathrm{~nm}$. The solution without sample was taken as control.

Antioxidant activity $(\%)=1-\frac{\operatorname{Abs}_{\text {Sample }}}{\operatorname{Abs}_{\text {Control }}} \times 100$

\subsection{Antimicrobial activity}

The synthesized Chi-Ag-NPs composites have pH of 4.3 and were adjusted to $\mathrm{pH}$ of 2.7. The $\mathrm{pH}$ of control (chitosan) was found to be 2.7. The antimicrobial activities of the synthesized Chi-Ag-NPs composites, silver nitrate salt and chitosan solution against pathogenic organisms were determined by agar disc diffusion method [1]. Gram positive bacteria such as Methicillin-resistant Staphylococcus aureus (MRSA) and gram negative bacteria such as $P$. aeruginosa and E.coli were chosen for the present study. The overnight cultured strain was individually swabbed on a solid Muller-Hinton agar (M-HA) medium in petri dish. $100 \mu$ l of synthesized Chi-Ag-NPs composites, silver nitrate salt and chitosan solution was transferred to the sterile disc placed on MHA petri plates. The antibiotic discs were placed over the plates and incubated for $24 \mathrm{~h}$ at $37^{\circ} \mathrm{C}$. The diameter of the zone of inhibition was expressed in millimeters. 


\subsection{Cytotoxicity}

Cytotoxicity/biocompatibility of Chi-Ag-NPs composites was investigated by MTT (3-(4, 5-dimethylthiazolyl-2)-2, 5-diphenyltetrazolium bromide) assay. This method is based on exposing viable cell to test sample in presence of MTT. The Chi-Ag-NPs composites were kept in 24 well plates and incubated with Dulbecco's modified eagle medium (DMEM) for $24 \mathrm{~h}$ to improve cell adhesion efficiency of Chi-Ag-NPs composites. A well without sample was served as control. After $24 \mathrm{~h}$, fibroblast cell suspension (in DMEM media) at a density of $5 \times 10^{4}$ cells $/ \mathrm{ml}$ and $100 \mu \mathrm{l}$ of MTT solution $(5 \mathrm{mg} / \mathrm{ml}$ ) were added to each well. The plate was placed at $37^{\circ} \mathrm{C}$ with $5 \% \mathrm{CO}_{2}$ atmosphere incubator for $3 \mathrm{~h}$ to allow the formation of formazan crystals in live cells. The pale yellow tetrazolium MTT is reduced by mitochondria of living cells to insoluble purple formazan crystals. The cells were vortexed well, centrifuged at $6000 \mathrm{rpm}$ for $2 \mathrm{~min}$ and then OD was measured at $540 \mathrm{~nm}$ in an ELISA microplate reader (SpecraMax M2e; Bucher Biotech, Basel, Switzerland). The intensity of colour developed is proportional to number of viable cells and was expressed in percentage (\%).

Cell viablity $=\frac{\mathrm{OD} \text { of sample }}{\mathrm{OD} \text { of control }} \times 100$

\section{Results and discussion}

\subsection{Characterization of Chi-Ag-NPs}

\subsubsection{UV-Vis absorption spectra and zeta potential of the Chi-Ag-NPs}

The synthesis of Chi-Ag-NPs was characterized by UV-visible spectroscopy. The UV-Vis absorption spectra of the synthesized Chi-Ag-NPs composite solution, chitosan solution and silver nanoparticles are depicted in Fig. 1. The phenomenon of change in colour of solution into red, green and brown indicate the formation of the particles. The colourless chitosan solution was changed to light yellow and then to yellowish brown which is an indicative of $\mathrm{Ag}^{+}$reduction. It is stated that the reducing agent reduce $\mathrm{Ag}^{+}$and lead to the formation of metallic silver and followed by agglomeration into oligomeric clusters. Eventually, these clusters lead to the formation of metallic colloidal silver nanoparticles (Ag-NPs) [2, 12]. In the present study, the biopolymer was used as protective agent to stabilize and protect the particle surfaces from agglomeration or losing their surface properties. The Chi-Ag-NPs
Table 1 Particle size and zeta potential value of chitosan and ChiAg-NPs

\begin{tabular}{llll}
\hline Sample & Size $(\mathrm{nm})$ & $\begin{array}{l}\text { Polydispersity } \\
\text { index (PDI) }\end{array}$ & Zeta potential \\
\hline Chitosan & $209 \pm 1.1$ & $0.310 \pm 0.01$ & $62.3 \pm 1.3$ \\
Chi-Ag-NPs (pH 4.3) & $233 \pm 0.5$ & $0.267 \pm 0.05$ & $78.3 \pm 0.6$ \\
\hline
\end{tabular}

composite exhibited peak at $419 \mathrm{~nm}$, is due to the excitation of surface plasmon resonance (SPR) of $\mathrm{AgNO}_{3}$ [13]. The characteristic SPR band of Chi-Ag-NPs composites was observed at $419 \mathrm{~nm}$, which is an indicative of interaction between amino and hydroxyl groups of chitosan polymer with silver ions [14]. Somee et al. [15] stated that SPR is a phenomenon related to energy equivalence between conductor electrons of metal and energy of electromagnetic waves irradiated to nanoparticles. No peak was observed in chitosan solution, due to absence of Ag-NPs (Fig. 9). It was stated that the concentration of silver salt influences the intensity of the SPR band as well as the absorbance of Ag-NPs. And it is also well known that SPR band of silver nanoparticles depends on the morphological shape, size, and distribution of nanoparticles [16]. According to Mie theory, nanoparticles with spherical shape have single SPR peak whereas the nanoparticles with asymmetric and different shapes have one or more shoulders in their SPR peak [17]. The increased absorbance/OD (optical density) values and change in $\mathrm{pH}$ of synthesized Chi-Ag-NPs defines the hyperchromic effect of $\mathrm{NaOH}$ and the role of $\mathrm{NaOH}$ signifies in nuclei formation and growth of silver nanoparticles [18].

Particle size measurements and Zeta potential of chitosan and synthesized Chi-Ag-NPs are given in Table 1 and Fig. 2. The particle size of chitosan and synthesized Chi-Ag-NPs had a Z-average diameter of $209 \mathrm{~nm}$ and

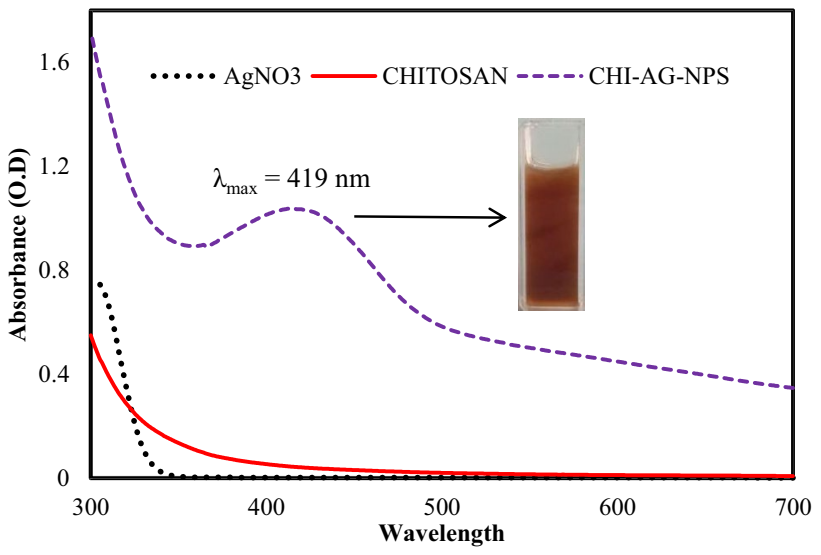

Fig. 1 UV-Vis absorption spectra of $\mathrm{AgNO}_{3}$, Chitosan and Chi-AgNPs 
$233 \mathrm{~nm}$ according to the size distributions in number in percentage with PDI of 0.310 and 0.267 . The silver nanoparticles grafted chitosan (Chi-Ag-NPs) have comparatively higher size than chitosan nanoparticles, this might be due to chelation of silver ions surface with chitosan polymer nanoparticles [19]. The particle size of the colloidal Ag-NPs could be regulated by changing the heating temperature and also by adjusting the ratio of biopolymer to the silver precursor [2]. As the $\mathrm{AgNO}_{3}$ concentration increases, the particle size of the silver nanoparticles increases and this results in the wider particle size distribution [20]. The PDI size distribution of Chi-NPs was $<1$. Furthermore, the detailed shape and morphological studies of the individual Chi-Ag-NPs composites were confirmed by SEM and TEM analysis.

Zeta potential is the potential that can be determined from particle mobility under an electric field. The mobility of particles depends on electrolyte concentration and surface charge. The zeta potential value of chitosan and Chi-Ag-NPs indicates that the surfaces have a positive charge of $62.3 \mathrm{mV}$ and $78.3 \mathrm{mV}$, and assumed that silver nanoparticles grafted chitosan suspension is stable in water due to electrostatic repulsion. The silver ions interaction takes place through the amino and hydroxyl groups of chitosan. The positively charged amine groups of chitosan a molecule which indicates partial protonation improves the electrostatic interaction with the negative charges of silver nanoparticles [6]. The difference in the zeta potential values might be due to the adsorption of chitosan and silver nanoparticle [21].

\subsubsection{Fourier transform infrared spectroscopy (FT-IR)}

FT-IR spectral analysis demonstrates the characteristics of surface structure and functional groups present in the reduction of $\mathrm{Ag}$ ions and the possible interaction between chitosan and Ag-NPs. The FT-IR spectra of chitosan and Chi-Ag-NPs are shown in Fig. 3. The spectral bands of chitosan at wave number $3375 \mathrm{~cm}^{-1}$ and $2881 \mathrm{~cm}^{-1}$ represents amide $A$ and amide $B$ bands. The amide $A$ arises mainly due to $\mathrm{O}-\mathrm{H}$ and $\mathrm{N}-\mathrm{H}$ stretching vibrations. The amide $B$ arises mainly due to the stretching vibrations of the aliphatic $-\mathrm{CH}$ bonds. Furthermore, the spectral bands at $1653 \mathrm{~cm}^{-1}, 1524 \mathrm{~cm}^{-1}$ and $1383 \mathrm{~cm}^{-1}$ arises due to $\mathrm{C}=\mathrm{O}$ stretching vibrations (Amide $\mathrm{I}$ ), $\mathrm{N}-\mathrm{H}$ bending vibrations (Amide II) and $\mathrm{CH} 2$ wagging vibrations (Amide III).

The FT-IR spectrum of Chi-Ag-NPs composites indicates a few alterations compared to chitosan. The amide A band of Chi-Ag-NPs was cleaved into two peaks at 3397 and $3164 \mathrm{~cm}^{-1}$. A new shoulder peak of Amide B band was appeared at $2926 \mathrm{~cm}^{-1}$. These changes might be due to the interactions of primary amino and amide groups between chitosan and Ag-NPs. The amide $\mathrm{A}$ and amide $B$ bands of Chi-Ag-NPs composites were not as broad as
Fig. 2 Particle size distribution of Chitosan and Chi-Ag-NPs
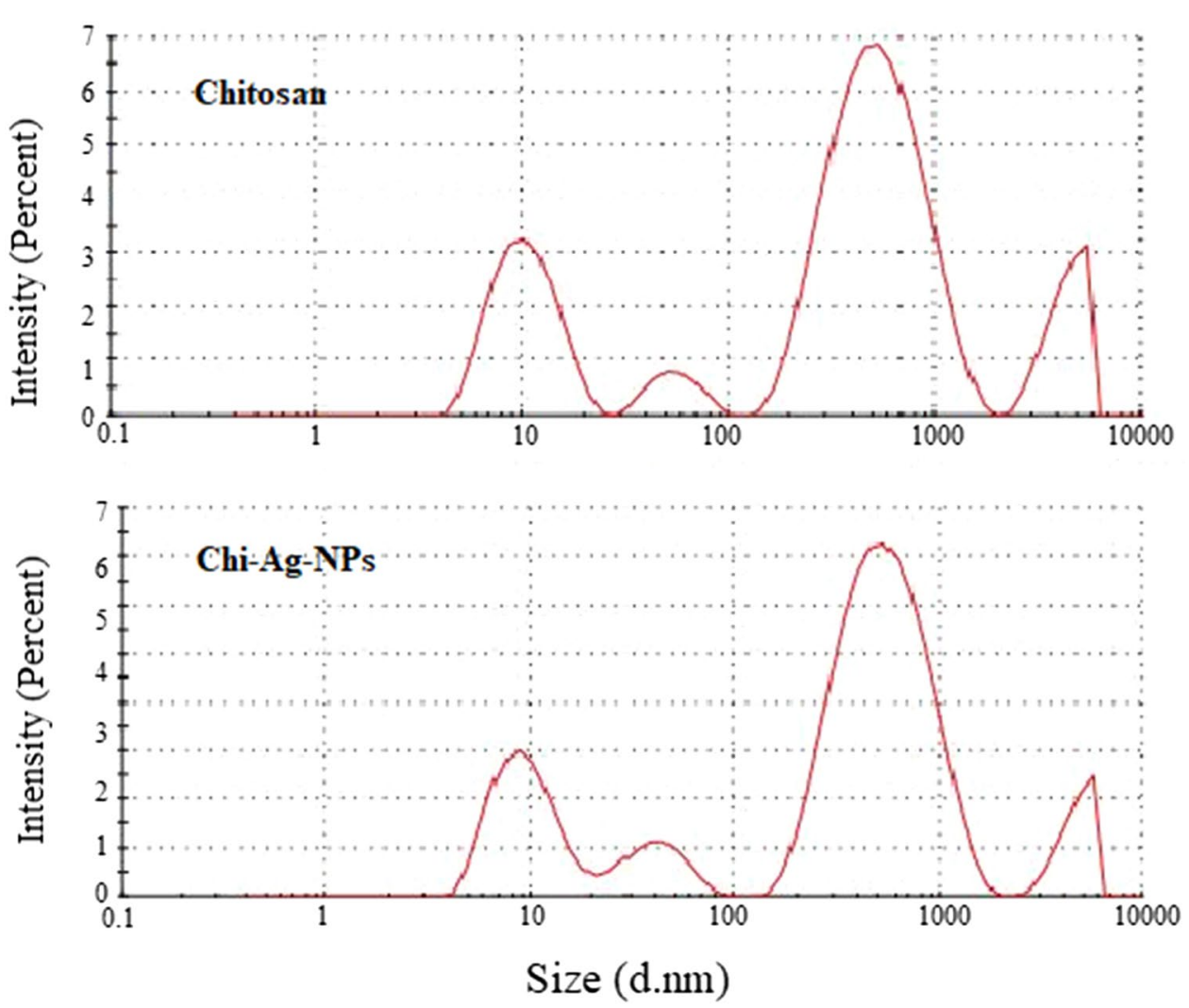
that of chitosan, which indicates the reduced hydrogen bonding [19]. In addition, the three amide bands I, II, and III of Chi-Ag-NPs composites were shifted to $1636 \mathrm{~cm}^{-1}$, $1557 \mathrm{~cm}^{-1}$ and $1399 \mathrm{~cm}^{-1}$ as well as the intensities of these three bands were decreased, which indicates the chelating of silver nanoparticles with chitosan amino and hydroxyl groups. Hajji et al. [22] stated that the presence of interactions between $\mathrm{Ag}, \mathrm{O}$ and $\mathrm{N}$ atoms of amide groups is also a reason for the shifting of wave numbers and lower intensities of peaks. The amide I band at $1636 \mathrm{~cm}^{-1}$ and $1653 \mathrm{~cm}^{-1}$ indicates that the interaction of synthesized silver nanoparticles (Ag-NPs) with polymer have not affected the secondary structure during the reaction [23]. Subsequently, the absorption peaks at $1072 \mathrm{~cm}^{-1}$ and $1083 \mathrm{~cm}^{-1}$ are attributed to C-O-C anti-symmetrical stretching linkage of polysaccharide moieties. The peaks observed in the range of $1000-450 \mathrm{~cm}^{-1}$ confirms the $\mathrm{CH}$ group and $-\mathrm{OH}$ out-of-plane deformation of Chi-AgNPs composites. All the five major bands of Chi-Ag-NPs composites indicate the formation of coordinate bonds between chitosan amino and hydroxyl groups and Ag-NPs.

\subsubsection{SEM}

The scanning electron micrographs of Chi-Ag-NPs with different magnification are depicted in Fig. 4A. Chi-Ag-NPs composites exhibit homogenous, dense, smooth, flake and porous surface structures indicating the Ag-NPs particles were aggregated and well distributed into the polymeric matrix. The formation of porous structures may be due to the reduction and stabilization process by chitosan polymeric chains [24]. This porous nature of composites is essential in antibacterial and biomedical application [25]. It revealed that the chitosan acts as an effective stabilizer which endorses the uniform distribution of Ag-NPs within the matrix.

\subsubsection{XRD}

The XRD technique was employed to find out the crystalline structure of the synthesized Chi-Ag-NPs and Chitosan polymer (Fig. 5). The characteristic peaks of chitosan was observed at $2 \theta=10.1^{\circ}$ and $2 \theta=19.9^{\circ}$. The peak at $2 \theta=19.9^{\circ}$ indicates the high degree of chitosan and their crystal lattice constant a corresponding to 4.4. The numbers of Bragg reflections of distinct peaks at $38^{\circ}, 44.1^{\circ}, 64^{\circ}$ and $77^{\circ}$ indicated the miller indices (111), (200), (220) and (311) reflections of metallic silver represent the face-centered cubic crystalline structure of silver [23]. A strong and sharp diffraction peak appeared at $38^{\circ}$ and $44.1^{\circ}$, can be indexed to the miller indices (111) and (200) reflection explains the cubic shape of the synthesized Chi-Ag-NPs as reported by Joint Committee on Power Diffraction Standards (JCPDS pdf no: 89-3722). The XRD pattern of Chi-Ag-NPs showed crystalline nature of the polymeric nanoparticles. Notably, no peaks of other impurity crystalline phases were observed.

\subsubsection{TEM}

The TEM micrographs of the synthesized Chi-Ag-NPs are presented in Fig. 4B. The TEM micrographs indicated that Ag-NPs are embedded in the chitosan nanocomposite matrix exhibiting spherical-like shape along with the particles of consistent geometry of pentagon and hexagon shape. It can be seen that Ag-NPs were distributed uniformly in the polymer sample. The silver nanoparticles grafted chitosan had a size range of $10-200 \mathrm{~nm}$ whereas,
Fig. 3 FTIR spectra of Chitosan and Chi-Ag-NPs

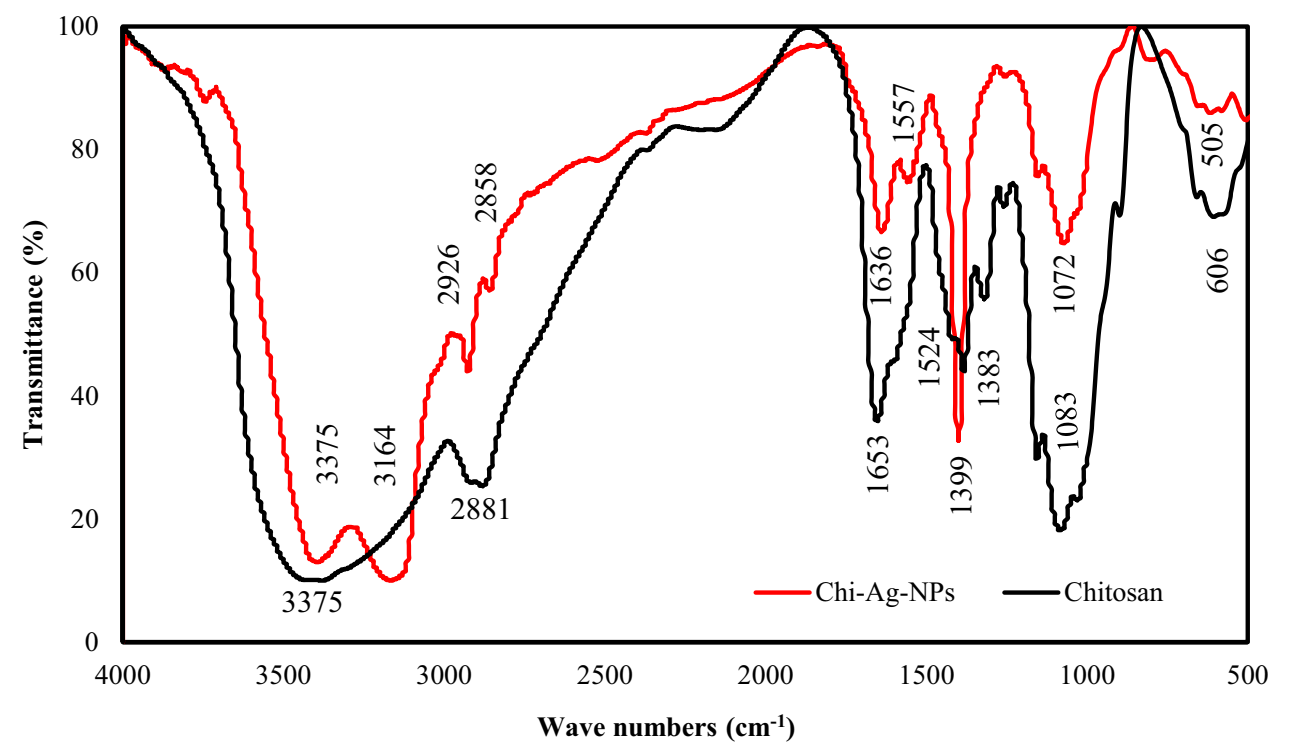


A
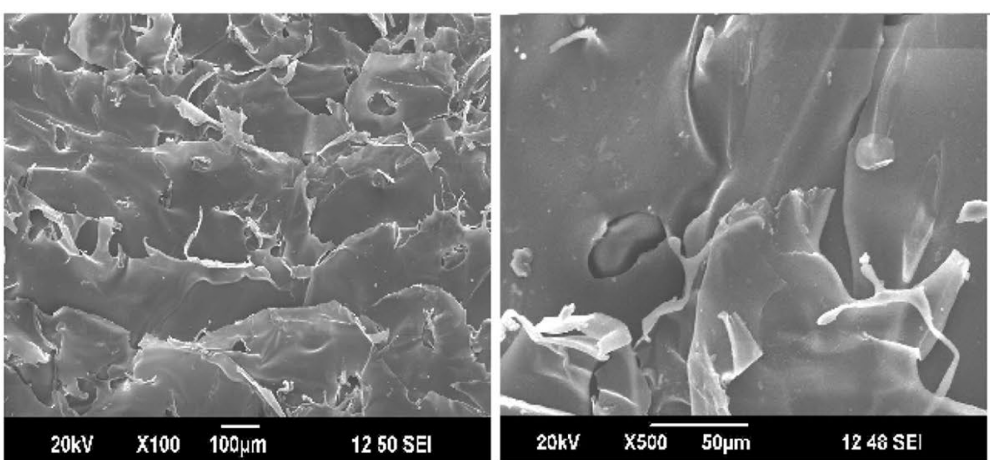

B
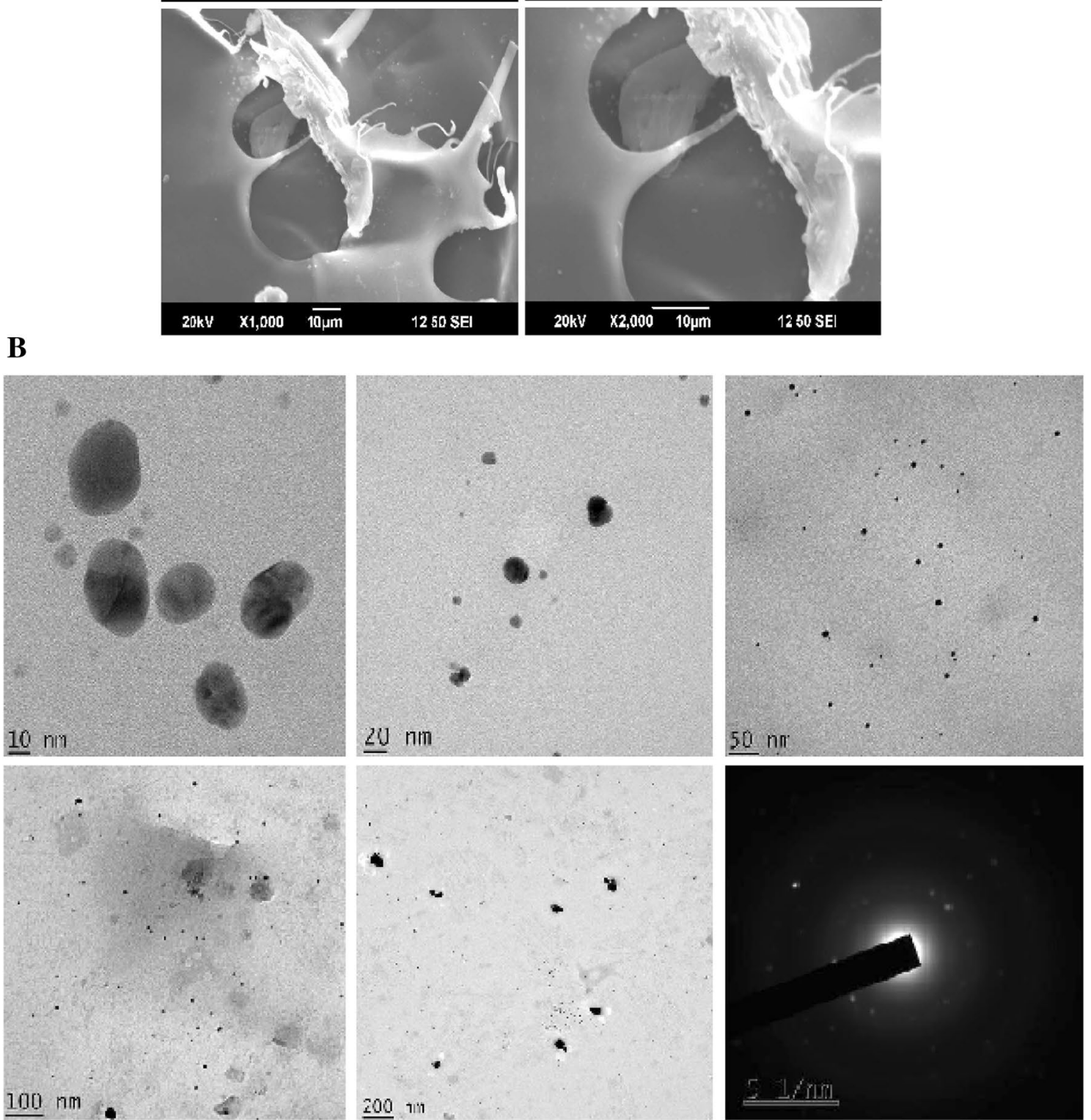

Fig. 4 A Scanning electron microscope (SEM) images of Chi-Ag-NPs. B TEM images of Chi-Ag-NPs

most of the particles formed had less than $10 \mathrm{~nm}$ size. Interestingly, it was found that the particles were reasonably uniform in size. The formation of increased size and different shape is due to the adsorption of silver on the surface of chitosan nanoparticles, which leads to form a chelate ring like structure. Kumar et al. [26] stated that 
the concentration, nature of stabilizing and reducing agents influences the size and shape of silver nanoparticles. The average size revealed by TEM micrographs is smaller for both chitosan nanoparticles and Chi-Ag-NPs as compared to that measured by Zeta sizer. This is due to the different principles and measurement conditions used. It was observed that the interaction between the chitosan polymer molecules and Ag-NPs either by coordinate or ionic bonds, promotes the dispersion of the silver nanoparticles without causing significant changes on the morphological properties [27].

\subsection{Antioxidant activity}

\subsubsection{Diphenyl-1-picryl hydrazyl activity (DPPH) assay}

The synthesized Chi-Ag-NPs composites were characterised for antioxidant properties by determining their DPPH radical scavenging ability. The DPPH solution which has purple colour turns to yellow on addition of Chi-Ag-NPs, which specifies the scavenging capability of free radicals and existence of antioxidant activity [28]. The radical scavenging activity of Chi-Ag-NPs increased from 6.77 to $60 \%(P<0.05)$ (Fig. 6A). The DPPH activity of the Chi-Ag-NPs was concentration-dependent. The DPPH activity increased with increase in concentration of Chi-Ag-NPs. The DPPH free radical was reduced by the Chi-Ag-NPs by donating or accepting electrons, which leads to the formation of hydrazine molecule [22]. The prepared Chi-Ag-NPs exhibiting antioxidant activity may be considered as proton-donators due to their primary amino and hydroxyl groups. It is also stated that the free amino and hydroxyl groups of chitosan has potential to react with free radicals which leads to form highly stable ammonium and molecular radical free groups [29].

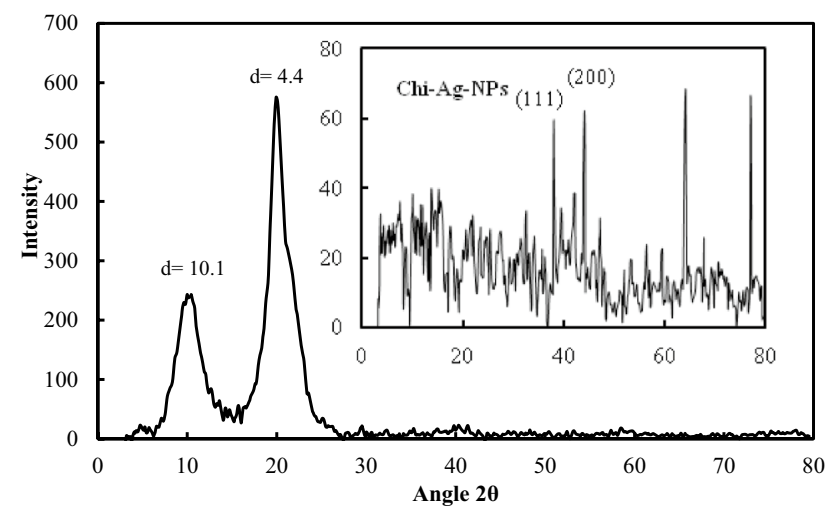

Fig. 5 XRD pattern of Chitosan and Chi-Ag-NPs

\subsubsection{Ferric reducing antioxidant power (FRAP) assay}

The ferric reducing power assay of synthesized Chi-Ag-NPs is presented in Fig. 6B. The reducing power of Chi-Ag-NPs was found to be in the range $0.15-0.62(P<0.05)$. The scavenging ability was noticed to be concentration dependent. These composites have ability to reduce the $\mathrm{Fe}^{3+}$ (ferric cyanide complex) to ferrous form. This $\mathrm{Fe}^{2+}$ complex can be examined by determining the Perl's Prussian blue at $700 \mathrm{~nm}$ [30]. It was observed that antioxidant activity and reducing power has direct correlation. The compounds have ability to donate electrons which exhibit higher reducing power. The formation of complexity between the hydroxyl and amino groups of chitosan with Ag ions, leads to decrease the binding sites for $\mathrm{Fe}^{2+}$ ions [9].

\subsection{3 $\beta$-Carotene-linoleic acid bleaching model system}

$\beta$-carotene-linoleic acid bleaching model system was used for the determination of antioxidant activity of synthesized Chi-Ag-NPs composites. The discoloration of $\beta$-carotene is considered to determine the antioxidant activity because $\beta$-carotene is most liable to free radicalmediated linoleic acid oxidation [31]. The antioxidant activity of Chi-Ag-NPs was in the range of $6.7-56.6 \%$ (Fig. 6A). The antioxidant activity of Chi-Ag-NPs increased with increase in concentration. The results revealed that the strong effects of synthesized Chi-Ag-NPs may act as natural antioxidants to protect against oxidative stress related with tissue engineering, wound healing and degenerative disease [6].

\subsection{Antimicrobial activity}

The antibacterial activity was exhibited by synthesized Chi-Ag-NPs composites against the gram negative and gram positive pathogens. The inhibition of tested pathogenic microorganism's growth indicates the antibacterial activity of nanocomposites (Fig. 7). The silver nanoparticles (Ag-NPs) are powerful antibacterial agents due to the interaction of silver $\left(\mathrm{Ag}^{+}\right)$ions with thiol (-SH) group of amino acids such as cysteine that are present in proteins. These proteins distinctively belong to respiratory enzyme class and this leads to inefficiency of respiratory system of bacterial cells and consequently cell death [32]. In the present study, the Chi-Ag-NPs $(\mathrm{pH}$ 2.7) exhibited the highest antibacterial activity against tested pathogens Methicillin-resistant Staphylococcus aureus (MRSA), E. coli and P. aeruginosa with $39 \mathrm{~mm}, 38 \mathrm{~mm}$ and $36 \mathrm{~mm}$ zone of inhibition respectively $(P<0.05)$ (Fig. 7). Interestingly, the silver nanoparticles grafted chitosan (Chi-Ag-NPs, pH 2.7) have highest antibacterial activity than the control (chitosan, $\mathrm{pH}$ 

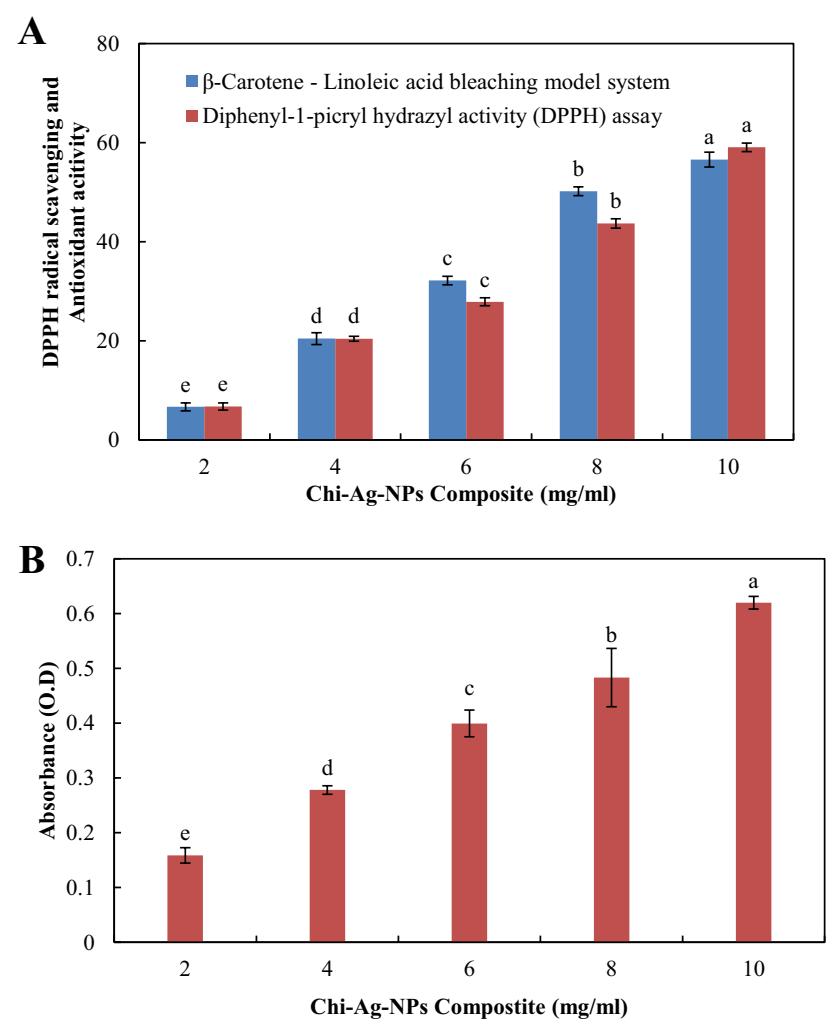

Fig. 6 A Diphenyl-1-picryl hydrazyl activity (DPPH) activity and $\beta$-Carotene-Linoleic acid bleaching model system. B Ferric reducing antioxidant power (FRAP) assay

2.7), silver nitrate salt and Chi-Ag-NPs (pH 4.3) $(P<0.05)$. Gram positive and gram negative bacteria usually are reported to have negative charge resulted from anionic properties of teichoic acids and lipopolysaccharides in their cell surface. The metal ions with positive charge can be adsorbed to the bacterial surface, results to cell wall disruption and inhibition of replication, respiratory function, and ATP production of bacteria. In contrast, the electrostatic interaction between positive charge of silver ions and negative charge of cell wall causes change in efflux and influx of biomaterials from bacteria [33]. Though the $\mathrm{pH}$ is same in control (chitosan, $\mathrm{pH} 2.7$ ) and synthesized Chi-Ag-NPs ( $\mathrm{pH}$ 2.7), the antibacterial activity is higher in Chi-Ag-NPs as compared to control, this might be due to the presence of Ag-NPs. Ag-NPs with nano scale are well known for more reactive and efficient in their antimicrobial activities due to high surface area [34]. The nanoparticles with lesser nanoscale can easily penetrate into the peptidoglycan layer of bacteria cell by forming hole and pit in cell membrane. Nanoparticles with less mean diameter size of 10-12 $\mathrm{nm}$ have more affinity to attach with thiol functional group of sulphurcontaining amino acids cysteine and methionine. This attachment may lead to change in NADH (nicotinamide adenine dinucleotide) dehydrogenases enzyme functionality of bacterial cell membrane [15].

Many researchers have studied the antibacterial activity of chitosan and Ag-NPs, but surprisingly, the antibacterial activity of chitosan grafted with Ag-NPs (Chi-Ag-NPs) is higher compared to that of chitosan and Ag-NPs components [35]. It is worthy to mention that several factors such as the nature and amount of reducing agent, type of metal salt, temperature, and $\mathrm{pH}$ have decisive effects on nanoparticles formation as well as governing the shape and size of synthesized nanoparticles [36]. It is also stated that the difference in antimicrobial activities might be due to the variation in the morphological shape, size, surface area of the nanoparticles and difference in structural and chemical compositions of bacterial cell membranes [37]. Comparatively, the bared $\mathrm{AgNO}_{3}$ had antibacterial effects similar to that of control chitosan. It was noticed that higher antibacterial activities of $\mathrm{AgNO}_{3}$ are resulted from intrinsic antimicrobial properties of $\mathrm{Ag}^{+}$ions [38].

\subsection{Cytotoxicity}

The Chi-Ag-NPs ( $\mathrm{pH}$ 2.7) which showed better antibacterial activity was subjected to biocompatibility studies. The in vitro cytotoxicity of Chi-Ag-NPs composites was investigated using the L929 fibroblast cell line by MTT assay and absorbance was recorded at $540 \mathrm{~nm}$ to evaluate cell viability. According to GB/T 16,886.5-2003 (ISO 10,993-5: 1999), the polymeric biomaterials with cell viability more than $75 \%$ can be considered as non-cytotoxic and are recommended for biomedical applications. In the present study, the cell viability of Chi-Ag-NPs composites was found to be $97 \%$ (Fig. 8A). No significant difference was observed in the cell viability between the Chi-Ag-NPs composites and the untreated control group $(P<0.05)$.

Figure $8 \mathrm{~B}$ demonstrates that fibroblast cells were adhered in a confluent monolayer over the surface of Chi-Ag-NPs composites and had the typical large, spindle shaped morphology. The adhesion, proliferation and growth of cells reveal the hydrophobic/hydrophilic balance, electrostatic interaction and biochemical functionalities of a biomaterial [39]. Theoretically, the extracellular matrix proteins with negative charge from the cell culture medium forms a thin layer by adsorption phenomenon, on the surface of polymeric matrix grafted with Ag-NPs with positive charge, this results in promoting the cell attachment and adhesion on composite biomaterial [40]. ROS (reactive oxygen species) production and oxidative stress are two notable and probable mechanisms of cytotoxic effects of nanoparticles on viable cells. The synthesized nanparticles interact with glutathione reductase enzyme, which binds to GSH and inhibits the activation of the antioxidant defence system and decrease GSH/ 
Fig. 7 Antimicrobial activity of Chi-Ag-NPs
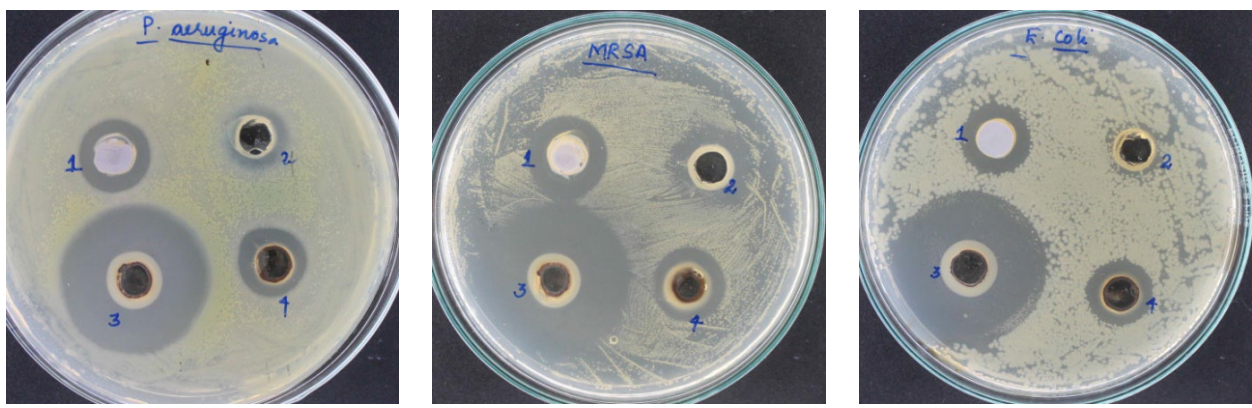

1- $\mathrm{AgNO}_{3}$; 2- Control (pH 2.7); 3- Chi-Ag-NPs (pH 2.7); 4- Chi-Ag-NPs (pH 4.3)

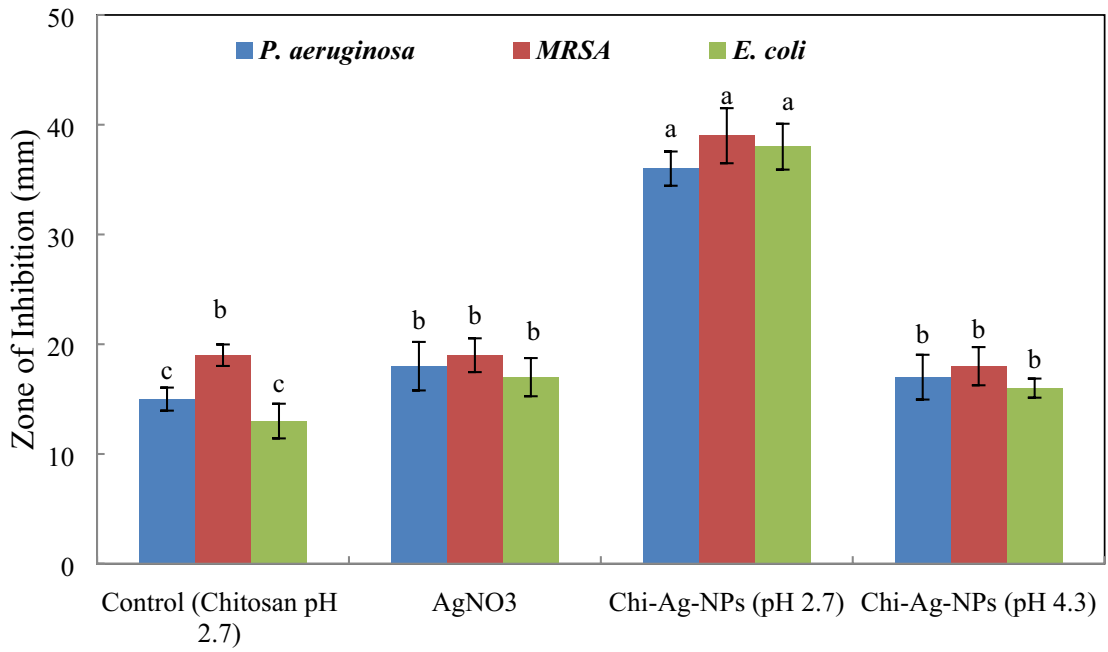

GSSG (glutathione/oxidized glutathione) ratio, resulting in the accumulation of ROS in the cells [15]. The growth of the fibroblast cells on the Chi-Ag-NPs composite membrane was found to be good, which emphasizes that silver nanoparticle grafted on chitosan polymer had no negative impact on the cell growth and cell proliferation. Therefore, synthesizing the biomaterial composites by grafting specific amount of Ag-NPs into polymeric matrix is prudent to reduce adequately bacterial adhesion and minimize tissue cytotoxicity [41]. The present results demonstrate that the synthesized Chi-Ag-NPs composites had good biocompatibility

\section{Conclusion}

Ag-NPs were synthesized by simple chemical method of using biodegradable chitosan as reducing agent. The physical, structural and morphological properties such as size, shape, dispersity of Chi-Ag-NPs were characterised by UV-Vis spectroscopy, Zeta potential, FTIR, SEM, TEM and XRD analysis. The synthesized Chi-Ag-NPs composites exhibited antioxidant properties as revealed by radical scavenging, reducing power and $\beta$-carotene-linoleic acid bleaching model system. In addition, the antibacterial

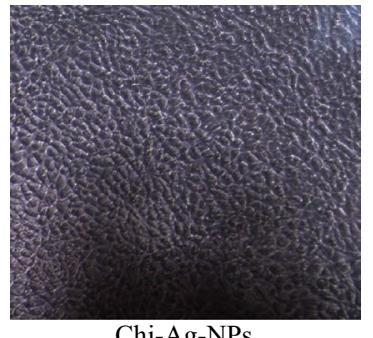

Chi-Ag-NPs
Fig. 8 A Cell Viability of Chi-Ag-NPs composites. B Cytotoxicity of Chi-Ag-NPs composites

\section{SN Applied Sciences}


activity was showed against selected pathogens viz gram positive Methicillin-resistant Staphylococcus aureus (MRSA), E. coli and gram nevative $P$. aeruginosa. The cell viability of Chi-Ag-NPs composites was found to be $97 \%$. From the results of present study, it can be concluded that Chi-AgNPs composites exhibit excellent bioactive, biocompatible and antibacterial properties and hence can be used as a bio-polymeric material in tissue engineering, biomedical and therapeutic applications.

Acknowledgements The authors would like to express their sincere gratitude to ICAR for providing funds to carry out the research work under ICAR-National Fellow Scheme. The authors acknowledge the Director, ICAR-Central Institute of Fisheries Technology (ICAR-CIFT), Cochin, Kerala, India for providing the facilities to carry out this work and also for granting permission to publish the data acquired from the study. The analytical services provided by "Sophisticated Test and Instrumentation Centre", Cochin University of Science and Technology, Cochin, Kerala are duly acknowledged. The authors are grateful to the Mrs. PA Jaya (Senior Technical Assistant), Mr. P. Suresh (Senior Technician), Mrs. N. Lekha (Technical Officer) ICAR-Central Institute of Fisheries Technology (CIFT), Cochin, Kerala for providing technical support to carry out the analyses.

\section{Compliance with ethical standards}

Conflict of interest The authors declare that they have no conflict of interest.

\section{References}

1. Manukumar HM, Umesha S, Kumar HN (2017) Promising biocidal activity of thymol loaded chitosan silver nanoparticles (TC@ AgNPs) as anti-infective agents against perilous pathogens. Int J Biol Macro 102:1257-1265

2. Iravani S, Korbekandi H, Mirmohammadi SV, Zolfaghari B (2014) Synthesis of silver nanoparticles: chemical, physical and biological methods. Res Pharm Sci 9(6):385

3. Venkatesan J, Lee JY, Kang DS, Anil S, Kim SK, Shim MS, Kim DG (2017) Antimicrobial and anticancer activities of porous chitosan-alginate biosynthesized silver nanoparticles. Int J Biol Macromol 98:515-525

4. Ali A, Ahmed S (2018) A review on chitosan and its nanocomposites in drug delivery. Int J Biol Macromol 109:273-286

5. Kalaivani R, Maruthupandy M, Muneeswaran T, Beevi AH, Anand M, Ramakritinan CM, Kumaraguru AK (2018) Synthesis of chitosan mediated silver nanoparticles (Ag NPs) for potential antimicrobial applications. Front Lab Med 2(1):30-35

6. Hajji S, Khedir SB, Hamza-Mnif I, Hamdi M, Jedidi I, Kallel R (1863) Nasri M (2019) Biomedical potential of chitosan-silver nanoparticles with special reference to antioxidant, antibacterial, hemolytic and in vivo cutaneous wound healing effects. Biochim et Biophysi Acta (BBA) Gen Subj 1:241-254

7. Wei D, Sun W, Qian W, Ye Y, Ma X (2009) The synthesis of chitosan-based silver nanoparticles and their antibacterial activity. Carbohydr Res 344(17):2375-2382

8. Chen Q, Jiang H, Ye H, Li J, Huang J (2014) Preparation, antibacterial, and antioxidant activities of silver/chitosan composites. J Carbohydr Chem 33(6):298-312
9. Yen GC, Wu JY (1999) Antioxidant and radical scavenging properties of extracts from Ganoderma tsugae. Food Chem 65(3):375-379

10. Oyaizu M (1986) Studies on products of browning reaction. Japn J Nutr Diet 44(6):307-315

11. Koleva II, Van Beek TA, Linssen JP, Groot AD, Evstatieva LN (2002) Screening of plant extracts for antioxidant activity: a comparative study on three testing methods. Phytochem Anal An Int J Plant Chem Biochem Tech 13(1):8-17

12. Ahmad A, Mukherjee P, Senapati S, Mandal D, Khan MI, Kumar R, Sastry M (2003) Extracellular biosynthesis of silver nanoparticles using the fungus Fusarium oxysporum. Colloids Surf B Biointerfaces 28(4):313-318

13. Jia X, Ma X, Wei D, Dong J, Qian W (2008) Direct formation of silver nanoparticles in cuttlebone-derived organic matrix for catalytic applications. Colloids Surf A Physicochem Eng Asp 330(2-3):234-240

14. Sanpui P, Murugadoss A, Prasad PD, Ghosh SS, Chattopadhyay A (2008) The antibacterial properties of a novel chitosan-Agnanoparticle composite. Int J Food Microbiol 124(2):142-146

15. Somee LR, Ghadam P, Abdi-Ali A, Fallah S, Panahi G (2018) Biosynthesised $\mathrm{AgCl}$ NPs using Bacillus sp. 1/11 and evaluation of their cytotoxic activity and antibacterial and anti-biofilm effects on multi-drug resistant bacteria. IET Nanobiotechnol 12(6):764-72

16. Wei D, Qian W (2008) Facile synthesis of Ag and Au nanoparticles utilizing chitosan as a mediator agent. Colloids Surf B: Biointerfaces 62(1):136-142

17. Kelly KL, Coronado E, Zhao LL, Schatz GC (2003) The optical properties of metal nanoparticles: the influence of size, shape, and dielectric environment. ACS Publications, Washington, D.C, pp 668-677

18. Yadav VD, Jain R, Dandekar P (2017) Influence of sodium hydroxide in enhancing the surface plasmon resonance of silver nanoparticles. Mater Res Express 4(8):085015

19. Ali SW, Rajendran S, Joshi M (2011) Synthesis and characterization of chitosan and silver loaded chitosan nanoparticles for bioactive polyester. Carbohydr Polyms 83(2):438-446

20. Cao XL, Cheng C, Ma YL, Zhao CS (2010) Preparation of silver nanoparticles with antimicrobial activities and the researches of their biocompatibilities. J Mater Sci Mater Med 21(10):2861-2868

21. Singare DS, Marella S, Gowthamrajan K, Kulkarni GT, Vooturi R, Rao PS (2010) Optimization of formulation and process variable of nanosuspension: an industrial perspective. Int J Pharm 402((1-2)):213-220

22. Hajji S, Salem RBSB, Hamdi M, Jellouli K, Ayadi W, Nasri M, Boufi $S$ (2017) Nanocomposite films based on chitosan-poly (vinyl alcohol) and silver nanoparticles with high antibacterial and antioxidant activities. Proc Saf Environ Prot 111:112-121

23. Das B, Dash SK, Mandal D, Ghosh T, Chattopadhyay S, Tripathy S, Roy S (2017) Green synthesized silver nanoparticles destroy multidrug resistant bacteria via reactive oxygen species mediated membrane damage. Arab J Chem 10(6):862-876

24. Vimala K, Mohan YM, Sivudu KS, Varaprasad K, Ravindra S, Reddy NN, MohanaRaju K (2010) Fabrication of porous chitosan films impregnated with silver nanoparticles: a facile approach for superior antibacterial application. Colloids Surf B Biointerfaces 76(1):248-258

25. Wang LS, Wang $C Y$, Yang $C H$, Hsieh $C L$, Chen $S Y$, Shen $C Y$, Huang KS (2015) Synthesis and anti-fungal effect of silver nanoparticles-chitosan composite particles. Int J Nanomed 10:2685

26. Kumar S, Shukla A, Baul PP, Mitra A, Halder D (2018) Biodegradable hybrid nanocomposites of chitosan/gelatin and silver nanoparticles for active food packaging applications. Food Packag Shelf Life 16:178-184 
27. Pinto RJ, Fernandes SC, Freire CS, Sadocco P, Causio J, Neto CP, Trindade T (2012) Antibacterial activity of optically transparent nanocomposite films based on chitosan or its derivatives and silver nanoparticles. Carbohydr Res 348:77-83

28. Zhang X, Geng X, Jiang H, Li J, Huang J (2012) Synthesis and characteristics of chitin and chitosan with the (2-hydroxy-3-trimethylammonium) propyl functionality, and evaluation of their antioxidant activity in vitro. Carbohydr Polym 89(2):486-491

29. Hari N, Francis S, Nair AGR, Nair AJ (2018) Synthesis, characterization and biological evaluation of chitosan film incorporated with $\beta$-Carotene loaded starch nanocrystals. Food Packag Shelf life 16:69-76

30. Chung YC, Chang CT, Chao WW, Lin CF, Chou ST (2002) Antioxidative activity and safety of the $50 \%$ ethanolic extract from red bean fermented by Bacillus Subtilis IMR-NK1. J Agric Food Chem 50(8):2454-2458

31. Lassoued I, Mora L, Nasri R, Jridi M, Toldrá F, Aristoy MC, Nasri M (2015) Characterization and comparative assessment of antioxidant and ACE inhibitory activities of thornback ray gelatin hydrolysates. J Funct Foods 13:225-238

32. Alavi M, Rai M (2019) Recent advances in antibacterial applications of metal nanoparticles (MNPs) and metal nanocomposites (MNCs) against multidrug-resistant (MDR) bacteria. Expert Rev Anti-infective Ther 17(6):419-428

33. Gold K, Slay B, Knackstedt M, Gaharwar AK (2018) Antimicrobial activity of metal and metal-oxide based nanoparticles. Adv Therapeutics 1(3):1700033

34. Gogoi SK, Gopinath P, Paul A, Ramesh A, Ghosh SS, Chattopadhyay A (2006) Green fluorescent protein-expressing escherichia c oli as a model system for investigating the antimicrobial activities of silver nanoparticles. Langmuir 22(22):9322-9328

35. Archana D, Singh BK, Dutta J, Dutta PK (2015) Chitosan-PVPnano silver oxide wound dressing: in vitro and in vivo evaluation. Int J Biol Macromol 73:49-57
36. Nayak RR, Pradhan N, Behera D, Pradhan KM, Mishra S, Sukla LB, Mishra BK (2011) Green synthesis of silver nanoparticle by Penicillium purpurogenum NPMF: the process and optimization. J Nanoparticle Res 13(8):3129-3137

37. Abdel-Mohsen AM, Jancar J, Abdel-Rahman RM, Vojtek L, Hyršl P, Dušková M, Nejezchlebová H (2017) A novel in situ silver/ hyaluronan bio-nanocomposite fabrics for wound and chronic ulcer dressing: in vitro and in vivo evaluations. Int J Pharm 520:241-325

38. Alavi M, Karimi N (2019) Biosynthesis of Ag and Cu NPs by secondary metabolites of usnic acid and thymol with biological macromolecules aggregation and antibacterial activities against multi drug resistant (MDR) bacteria. Int J Biol Macromol 128:893-901

39. Liu BS, Huang TB (2008) Nanocomposites of genipin-crosslinked chitosan/silver nanoparticles- structural reinforcement and antimicrobial properties. Macromol Biosci 8(10):932-941

40. Brynda E, Pacherník J, Houska M, Pientka Z, Dvořák P (2005) Surface immobilized protein multilayers for cell seeding. Langmuir 21(17):7877-7883

41. Anitha A, Rani VD, Krishna R, Sreeja V, Selvamurugan N, Nair SV, Jayakumar R (2009) Synthesis, characterization, cytotoxicity and antibacterial studies of chitosan, O-carboxymethyl and N O-carboxymethyl chitosan nanoparticles. Carbohydr Polym 78(4):672-677

Publisher's Note Springer Nature remains neutral with regard to jurisdictional claims in published maps and institutional affiliations. 